\title{
Forgiveness, reconciliation and shame: Three key variables in reducing school bullying
}

\author{
Dr Eliza Ahmed \\ Centre for Restorative J ustice \\ Australian National University \\ http://www.crj.anu.edu.au
}




\section{Bullying is}

- a repetitive aggressive act (either physical or non - physical)

- the dominance of the powerful(s) over the powerless(s) who is or are not capable of retaliating

- carried out without provocation 


\section{What does past research tell us?}

\section{Four main research traditions:}

- Nature and prevalence

- Family variables

- School variables

- Individual characteristics

(Sources: Ahmed, 2001; Bowers, Smith, \& Binney, 1994; Espelage, Bosworth, \& Simon, 2000; Olweus, Limber, \& Mihalic, 1999; Rigby \& Cox, 1996; Rigby, Cox, \& Black, 1997; Shields \& Cicchetti, 2001; Slee, 1993) 


\section{What is missing from past research?}

- A restorative justice perspective

- Forgiveness and reconciliation

- Emotions (e.g., shame / guilt)

- Non-western cultural context 
Hypothesis 1

Forgiveness reduces bullying behavior

Hypothesis 2

Reconciliation reduces bullying behavior 


\section{Shame and its management}

SHAME ACKNOWLEDGMENT (adaptive)

- feeling shame

- taking responsibility

- making amends

SHAME DISPLACEMENT (non-adaptive)

- blaming others

- hitting out at others

- feeling retaliatory anger 


\section{Hypothesis 3}

Shame acknowledgment reduces bullying

Hypothesis 4

Shame displacement triggers bullying 


\section{Mediational hypothesis (via shame management variables)}

Forgiveness

Figure 1. A hypothesized model of forgiveness, reconciliation, shame management and bullying (Model A) 


\title{
An alternative Mediational hypothesis (via forgiveness and reconciliation)
}

\author{
Forgiveness
}

\section{Reconciliation}

Figure 2. An alternative hypothesized model of shame management, forgiveness, reconciliation and bullying (Model B) 


\section{Methodology}

- Data collected through the "Life at School Survey" (Bangladesh, South Korea, and Australia)

- 1875 students from Dhaka, Bangladesh

- Recruited from 9 co-educational schools, both public and private

- Female - $60 \%$

- average school grade - 8.28 
Table 1. Correlation coefficients between school bullying and predictor variables

Variables

Correlation coefficients

Forgiveness

Reconciliation

Shame acknowledgment

Shame displacement
$-.38 * * *$

$-.67 * * *$

$-.20 * * *$

$.27 * * *$

*** $p<.001$ 


\section{Figure 3. Results of a path analysis (Model A)}

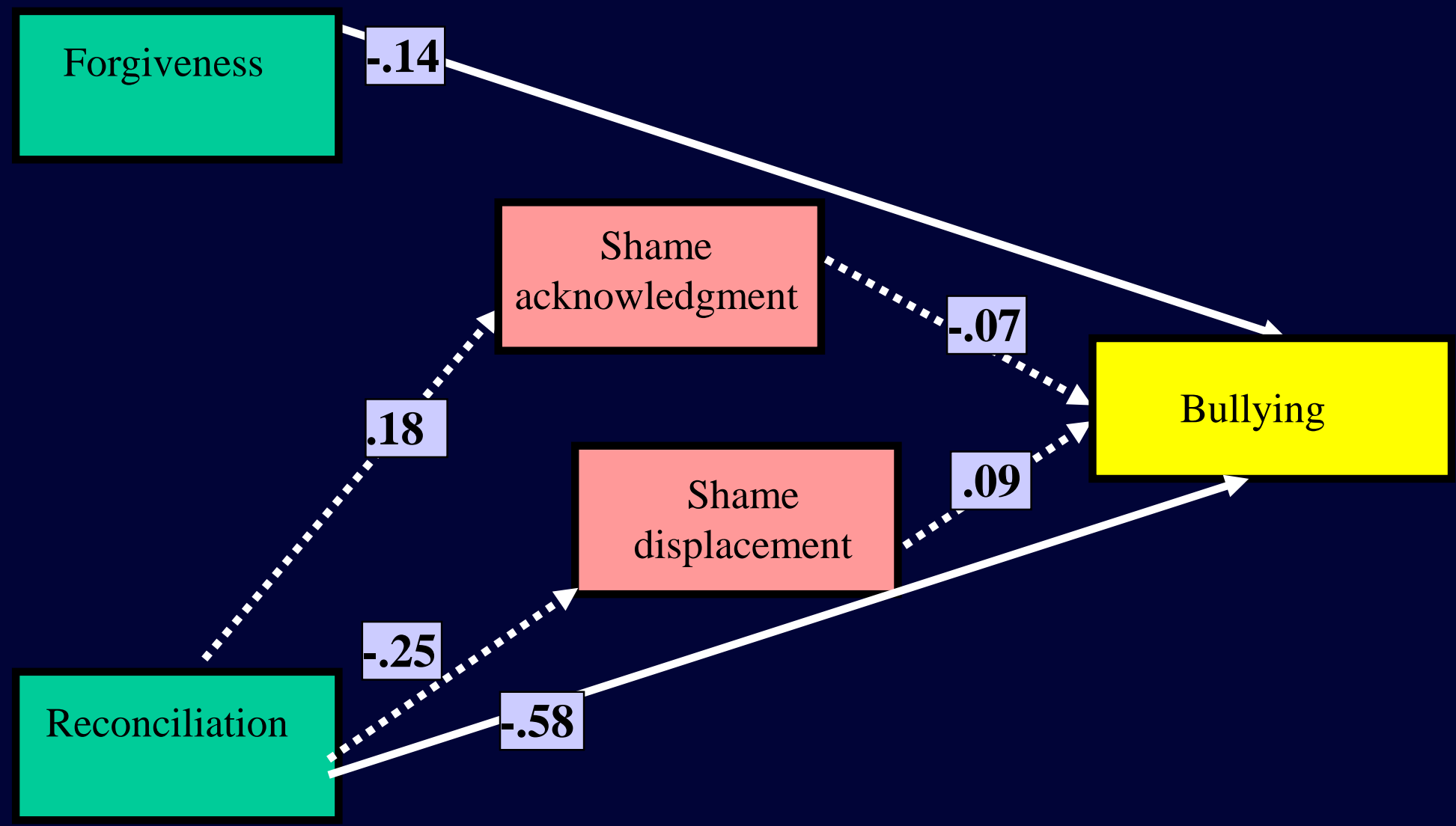




\section{Figure 4. Results of a path analysis (Model B)}

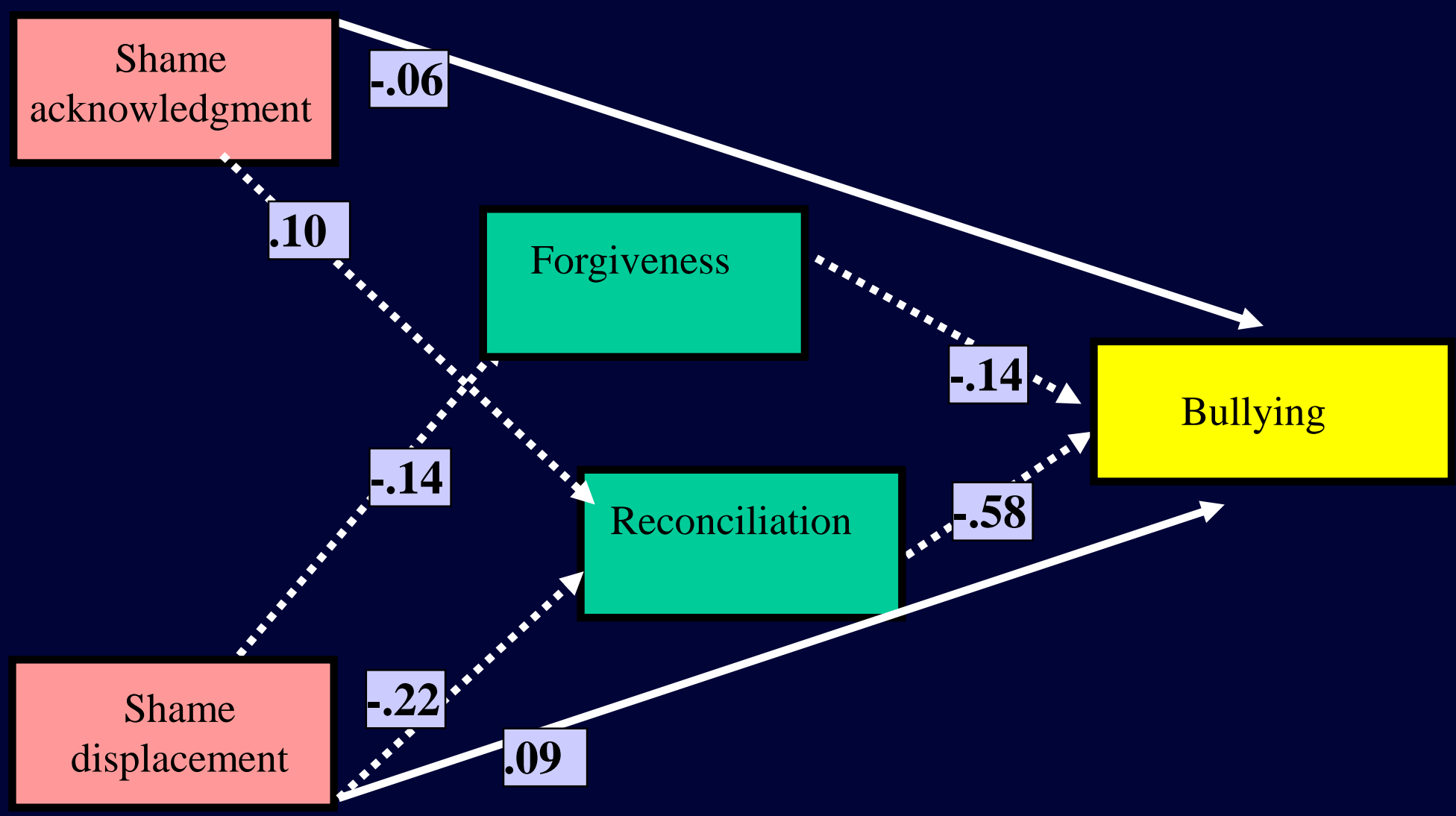




\section{Table 2. A comparison between two path models}

\begin{tabular}{lcc} 
Goodness-of-fit indices & Model A & Model B \\
\hline Chi-square $\left(\chi^{2}\right)$ & $1.99(\mathrm{df}=2 ; \mathbf{p}<.37)$ & $.14(\mathrm{df}=1 ; \mathbf{p}<.71)$ \\
GFI (Goodness of Fit Index) & .999 & 1.00 \\
AGFI (Adjusted Goodness of Fit Index) & .995 & .998 \\
CFI (Comparative Fit Index) & 1.00 & 1.00 \\
TLI (Tucker-Lewis Index) & 1.00 & 1.00 \\
RMSEA (Root Mean Square Error of Approximation) & .000 & .000 \\
AIC (Akaike's Informational Criterion) & 27.98 & 28.14 \\
CAIC (Consistent Akaike's Informational Criterion) & 106.63 & 112.84 \\
ECVI (Expected Cross-Validation Index) & .02 & .02 \\
\hline
\end{tabular}




\section{Summary of results}

- Forgiveness reduces bullying

- Reconciliation reduces bullying

- Shame acknowledgment reduces bullying

- Shame displacement triggers bullying 